\title{
HTR7 promotes laryngeal cancer growth through PI3K/AKT pathway activation
}

\author{
Xiaoli Sheng ${ }^{1,2 \#}$, Wenlin Liu ${ }^{2,3 \#}$, Zhongming Lu ${ }^{1,2}$, Mimi Xu ${ }^{1}$, Rui Li ${ }^{1,4}$, Rong Zhong ${ }^{1,4}$, Yunxian $\mathrm{Li}^{1,5}$, \\ Tao Liu ${ }^{1}$, Siyi Zhang ${ }^{1,2}$
}

${ }^{1}$ Department of Otorhinolaryngology, Guangdong Provincial People's Hospital and Guangdong Academy of Medical Sciences, Guangzhou, China; ${ }^{2}$ Second School of Clinical Medicine, Southern Medical University, Guangzhou, China; ${ }^{3}$ Department of Otorhinolaryngology, The Sixth Affiliated Hospital of Guangzhou Medical University, Qingyuan People's Hospital, Qingyuan, China; ${ }^{4}$ South China University of Technology, Guangzhou, China; ${ }^{5}$ Shantou University Medical College, Shantou, China

Contributions: (I) Conception and design: S Zhang, T Liu; (II) Administrative support: None; (III) Provision of study materials or patients: Y Li; (IV) Collection and assembly of data: X Sheng, W Liu, Z Lu, M Xu, R Li; (V) Data analysis and interpretation: X Sheng, W Liu, Z Lu, M Xu, R Li; (VI) Manuscript writing: All authors; (VII) Final approval of manuscript: All authors.

"These authors contributed equally to the work.

Correspondence to: Tao Liu; Siyi Zhang. Department of Otorhinolaryngology, Guangdong Provincial People's Hospital and Guangdong Academy of Medical Sciences, No. 106 Zhongshan Er Road, Guangzhou 510080, China. Email: taoliu18@126.com; szhang555@hotmail.com.

Background: Laryngeal cancer is a common malignancy of the head and neck, it's important to find novel targets for its therapy. The 5-hydroxytryptamine receptor 7 (HTR7) belongs to the G protein-coupled receptors (GPCRs) family which are easily druggable in diseases; however, its role in laryngeal cancer remains unknown.

Methods: Colony formation assay, Soft agar growth assay, BrdU incorporation assay and MTT assay were used to analyze the effect of HTR7 on laryngeal cancer cell proliferation. Xenograft tumors in nude mice was used to analyze the effect of HTR7 on laryngeal cancer growth. Luciferase reporter assay was used to analyze the effect of HTR7 on phosphatidylinositol-4,5-bisphosphate 3-kinase (PI3K)/protein kinase B (AKT) pathway activity.

Results: We found that HTR7 was significantly upregulated in laryngeal cancer tissues and cells, and patients with high HTR7 expression had shorter survival time than those with low HTR7 expression. Univariate and multivariate Cox regression models showed that HTR7 was an independent predictive factor for the prognosis of patients with laryngeal cancer. Cell proliferation assays and an animal model showed that HTR7 overexpression promoted laryngeal cancer proliferation and growth, while HTR7 knockdown inhibited laryngeal cancer proliferation and growth. Further analysis showed HTR7 activated the PI3K/AKT pathway, characterized by increased phosphorylation of AKT, luciferase reporter activity of forkhead box O (FOXO) factors, and target expression. Inhibition of the PI3K/AKT pathway in HTR7-overexpressing cells suppressed proliferation and growth, suggesting that HTR7 promotes laryngeal cancer proliferation and growth by activating the PI3K/AKT pathway.

Conclusions: HTR7 is not only a target for laryngeal cancer therapy but also a prognostic factor for the prognosis of patients with laryngeal cancer.

Keywords: 5-hydroxytryptamine receptor 7; laryngeal cancer; protein kinase B (AKT); tumor growth

Submitted Jan 21, 2021. Accepted for publication Apr 28, 2021.

doi: 10.21037/atm-21-1069

View this article at: http://dx.doi.org/10.21037/atm-21-1069 


\section{Introduction}

Laryngeal cancer is a common malignancy of the head and neck, with approximately 11,000-13,000 cases of laryngeal cancer being diagnosed annually in China (1). In the past decade, laryngeal cancer treatment has improved with surgery, radiation, systemic therapy, and novel agents for metastasis (2); however, the death rate is still high. Therefore, to improve laryngeal cancer therapy, a better understanding of the regulatory mechanism of laryngeal cancer is urgently required.

The phosphatidylinositol-4,5-bisphosphate 3-kinase $(\mathrm{PI} 3 \mathrm{~K}) /$ protein kinase B (AKT) pathway is frequently mutated in human cancers, and several PI3K families exist in human cells (3). Class IA PI3Ks, which are known to regulate tumor progression, are heterodimers consisting of a p85 regulatory subunit and a p110 catalytic subunit. Growth factor receptor tyrosine kinases, such as (EGFR), HER2, MET, and FGFR, phosphorylate adaptor proteins to bind $\mathrm{p} 85$, which relieves the inhibition of $\mathrm{p} 110$ by $\mathrm{p} 85$. The $\mathrm{p} 85$-p 110 heterodimer then binds to lipid phosphatidylinositol-4,5-bisphosphate (PIP2) at the plasma membrane. p110 phosphorylates PIP2 to generate phosphatidylinositol-3,4,5-triphosphate (PIP3). PTEN and INPP4B can dephosphorylate PIP3 to inhibit the PI3K/AKT pathway. AKT, SGK, and PDK1 bind to PIP3 at the plasma membrane, and then PDK1 and TORC2 phosphorylate AKT to fully activate AKT. Activated AKT can phosphorylate many proteins involved in cell survival, proliferation, growth, and protein synthesis, such as FOXO family proteins, MDM2, and p27 (4,5).

5 -hydroxytryptamine (serotonin) receptor 7 (HTR7) is a G protein-coupled receptor (GPCR) first described in 1993, and many transcription factors can bind to its promoter, such as AP2, EGR-1, and MAZ (6,7). HTR7 is associated with circadian rhythm, anxiety, depression, schizophrenia, nociception, and memory (8-10). However, the role of HTR7 in tumor progression has not been studied. In the present study, we aimed to investigate the role of HTR7 in laryngeal cancer. The findings indicated that HTR7 promotes laryngeal cancer growth by activating the AKT pathway. We present the following article in accordance with the ARRIVE reporting checklist (available at http:// dx.doi.org/10.21037/atm-21-1069).

\section{Methods}

\section{Cell culture and specimens}

Human oral keratinocytes cell and laryngeal cancer cell
TU212, FaDu, Hep-2, TU212, and TU686 were purchased from the American Type Culture Collection (ATCC) and cultured in RPMI 1640 medium (Invitrogen, Carlsbad, CA, USA) supplemented with $10 \%$ fetal bovine serum (FBS, Biological Industries, Kibbutz Beit-Haemek, Israel) at $37^{\circ} \mathrm{C}$ in a humidified incubator with $5 \% \mathrm{CO}$. All laryngeal cancer tissues and adjacent normal laryngeal tissues were obtained from LSCC patients who underwent surgery at the Otolaryngology Head and Neck Department of Guangdong Provincial People's Hospital (Guangzhou, Guangdong, China). Eight pairs of fresh laryngeal cancer tissues and adjacent normal laryngeal tissues were obtained during the procedures and immediately frozen in liquid nitrogen. A total of 113 laryngeal cancer tissues were obtained; the detailed clinicopathological characteristics are shown in Table S1. The study was conducted in accordance with the Declaration of Helsinki (as revised in 2013). The Ethics Committee of Guangdong Provincial People's Hospital and Guangdong Academy of Medical Sciences approved all collections. All patients provided written informed consent.

\section{Quantitative reverse transcription polymerase chain reaction ( $q R T-P C R)$}

Total RNA was isolated using the FastPure Cell/Tissue Total RNA isolation Kit (Vazyme, Nanjing, Jiangsu, China) according to the manufacturer's protocols. RNA was reversely transcribed into cDNA according to the instructions of the HiScript III 1st Strand cDNA Synthesis Kit (+ gDNA wiper; Vazyme). qRT-PCR was performed on the CFX-96 PCR system (Bio-Rad) using the AceQ Universal SYBR qPCR Master Mix (Vazyme). The relative quantification of mRNA expression was calculated using the $2^{-\Delta \Delta \mathrm{Ct}}$ method. GAPDH was used as the negative control for detecting mRNA expression.

\section{Western blotting}

Total protein was extracted from cells and tissues using RIPA buffer supplemented with protease inhibitor cocktail (Sigma, St. Louis, MI, USA), protein concentration was determined using the Pierce BCA Protein Assay Kit (Thermo Fisher Scientific, Waltham, MA, USA). Proteins were separated by sodium dodecylsulfate-polyacrylamide gel electrophoresis and transferred to polyvinylidene difluoride membranes. The membranes were blocked using $5 \%$ skimmed milk at room temperature for $1 \mathrm{~h}$. The membranes were then incubated with primary antibodies at 
$4{ }^{\circ} \mathrm{C}$ overnight, and incubated with horseradish peroxidaselabeled secondary antibodies. Finally, the membranes were immersed in electrochemiluminescence luminescence and exposed using a chemiluminometer. The primary antibodies were as follows: HTR7 (ab137493; Abcam, Cambridge, UK), p21 (\#2947, CST, Danvers, MA. USA), p16 (\#80772, CST), cyclin D1 (\#55506, CST), cyclin E (\#81045, CST), AKT (\#9272, CST), p-AKT (\#9611, CST), and GAPDH (\#5174, CST).

\section{Small molecule compound, vector construction, siRNA synthesis, and transfection}

AKT inhibitor, perifosine (s1037), was purchased from Selleck and dissolved using DMSO (Sigma). siAKT (siG150820105010-1-5) was purchased from RiBoBio (Guangzhou, Guangdong, China). To overexpress HTR7, the CDS sequence was cloned into lentivirus vector pSin-EF2-Puro; to knockdown HTR7, 2 shRNA sequences were cloned into lentivirus vector PLKO.1-pur. The shRNA sequences were: shHTR7\#1: 5'-GCACACCAACAGAACTGAGTT-3' and shTHR7\#2: 5'-CCAGGACTTTGGCTATACGAT-3'. The vectors were co-transfected with packing vectors pSPAX2 and pM2. G into 293FT using Lipofectamine 3000 (Thermo Fisher Scientific, USA) to generate lentivirus. The lentivirus were infected cells for overnight; stable cell lines were screened using Puromycin (Sigma).

\section{3-(4,5-Dimethylthiazol-2-yl)-2,5-diphenyltetrazolium bromide (MTT)}

Indicated cells were seeded in a 96-well plate at a density of $4 \times 10^{3}$ cells/well, six replications were prepared for the treatment group. Cells were incubated with MTT solution at the indicated time points for $4 \mathrm{~h}$ at $37^{\circ} \mathrm{C}$; DMSO was added to each well. The plates were gently shaken on a horizontal shaker for $10 \mathrm{~min}$ to dissolve crystals; the optical density value of each well was read at $570 \mathrm{~nm}$ in a microplate reader (BioTek). Each experiment was repeated 3 times.

\section{Colony formation assay and BrdU incorporation assay}

Cells were seeded in 6-well plates at a density of $0.5 \times 10^{3}$ cells/well and cultured for 10 days. Colonies were fixed with $10 \%$ formaldehyde for $5 \mathrm{~min}$ and then stained with $1 \%$ crystal violet for $30 \mathrm{~s}$. BrdU incorporation assay was performed using the BrdU Kit (eBioscience, San Diego, CA, USA).

\section{Soft agar growth assay}

Cells were resuspended in $2 \mathrm{~mL}$ complete medium plus $0.3 \%$ agar. The agar-cell mixture was plated on the top of a bottom layer consisting of $1 \%$ agar in complete medium. Ten days later, colony size was measured using an ocular micrometer, and colonies $>0.1 \mathrm{~mm}$ in diameter were counted. The experiment was performed 3 times for each cell line.

\section{Xenograft tumors in nude mice}

All animal experiments were performed in accordance with the guidelines for animal care and under the protocols approved by the Institutional Animal Care and Use Committee of the Guangdong Provincial People's Hospital and Guangdong Academy of Medical Sciences. Nude mice (4 weeks old) were purchased from the Model Animal Research Center of Nanjing University and randomly assigned into the following 4 groups, with 3 mice in each group: the vector control group, the HTR7 overexpression group, the scramble group, and the HTR7 knockdown group. The dorsal flank of each nude mouse was injected subcutaneously with $1 \times 10^{7}$ cells. The length $(\mathrm{L})$ and width (W) of the tumors were measured with a Vernier caliper. The volume of the tumor was calculated as Volume $(\mathrm{V})=$ $W^{2} \times L \times 0.52$. Tumor size were measured weekly.

\section{Statistical analysis}

All data were processed using SPSS version 21.0 (IBM, Armonk, NY, USA). Measured data were expressed as mean \pm standard deviation. Comparisons between 2 groups were conducted with independent $t$-test. One-way analysis of variance was used to compare difference in multiple groups. $\mathrm{P}<0.05$ indicated statistical significance.

\section{Results}

\section{HTR7 is upregulated in laryngeal carcinoma tissues and cells}

We found that HTR7 was significantly upregulated in laryngeal cancer tissues compared with that in normal laryngeal tissues in data from The Cancer Genome Atlas 


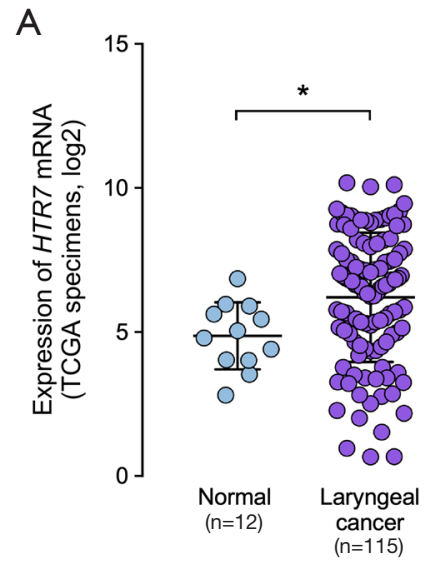

D

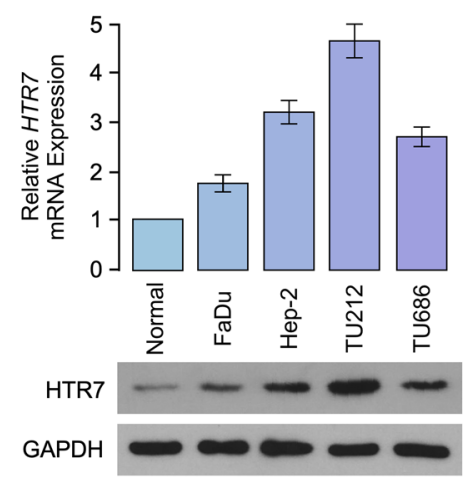

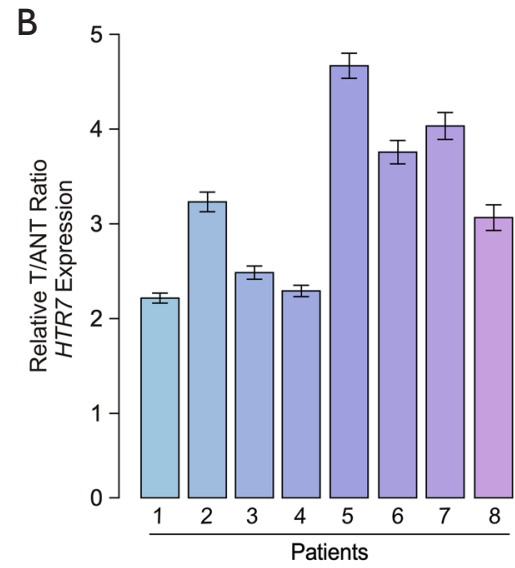

E

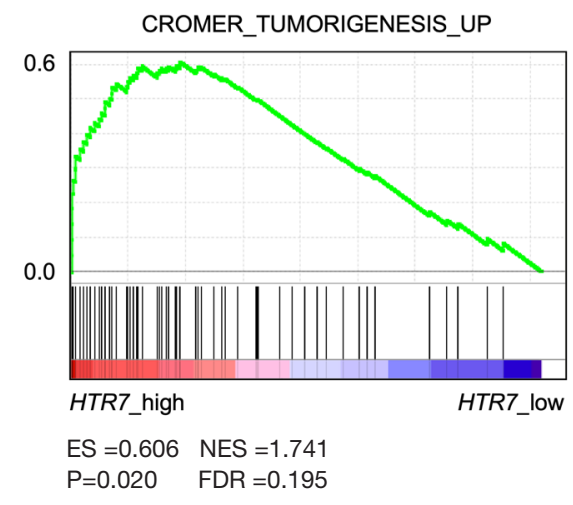

C

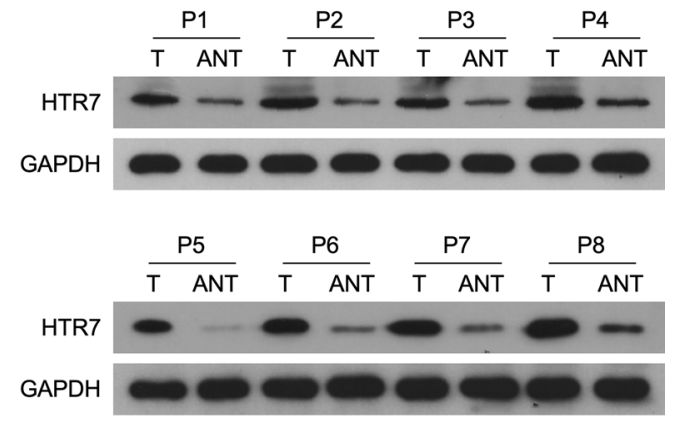

F

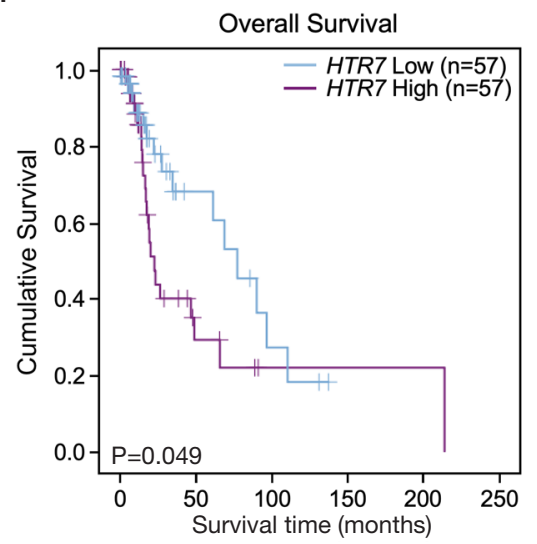

Figure 1 5-hydroxytryptamine receptor 7 (HTR7) is overexpressed in laryngeal cancer tissues and cells. (A) HTR7 was significantly upregulated in laryngeal cancer tissues compared with that in normal laryngeal tissues; data were downloaded from The Cancer Genome Atlas. (B) Quantitative reverse transcription polymerase chain reaction (qRT-PCR) analysis of HTR7 expression in laryngeal cancer tissues (T) and adjacent normal tissues (ANT). (C) Western blot analysis of HTR7 levels in laryngeal cancer tissues (T) and adjacent normal tissues (ANT). GAPDH was used as the loading control. (D) qRT-PCR and Western blot analysis of HTR7 expression in laryngeal cancer cells and normal laryngeal epithelial cells. GAPDH was used as the loading control for the Western blot analysis. (E) Gene Set Enrichment Analysis of the relationship between HTR7 expression levels and tumorigenesis. (F) Survival curve analysis of HTR7 expression and clinical outcome. Error bars represent the (SEM). ${ }^{*} \mathrm{P}<0.05$.

(TCGA) database (Figure 1A). We also confirmed this result using 8 pairs of laryngeal cancer tissues and their adjacent normal tissues: qRT-PCR and Western blotting showed that HTR7 was also upregulated in laryngeal tissues (Figure $1 B, C$ ). qRT-PCR and Western blotting further demonstrated that HTR7 was upregulated in laryngeal cells compared with that in normal laryngeal epithelia cells (Figure 1D). Gene Set Enrichment Analysis (GSEA) showed the high HTR7 expression was positively correlated with strong tumorigenesis ability (Figure 1E). Finally, a further analysis of TCGA data revealed that patients with high
HTR7 expression had a shorter survival time than those with low HTR7 expression (Figure 1F). Taken together, these findings suggested that HTR7 was upregulated in laryngeal cancer tissues and cells, and high HTR7 expression was associated with poor outcome.

\section{HTR7 is an independent predictive factor for the prognosis of patients with laryngeal cancer}

On the basis of these results, we determined the association between HTR7 expression and the overall survival of 
A

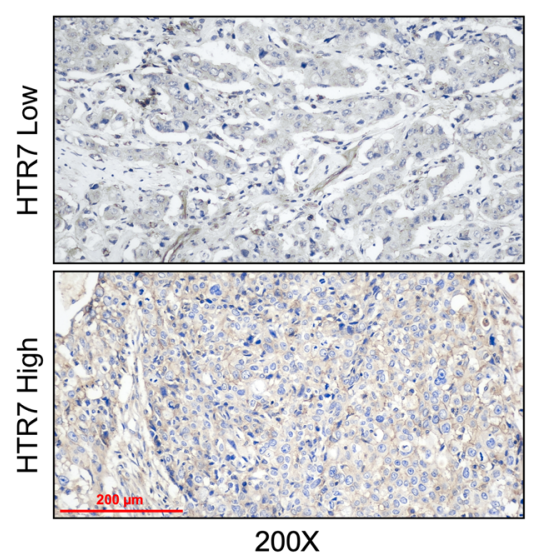

B

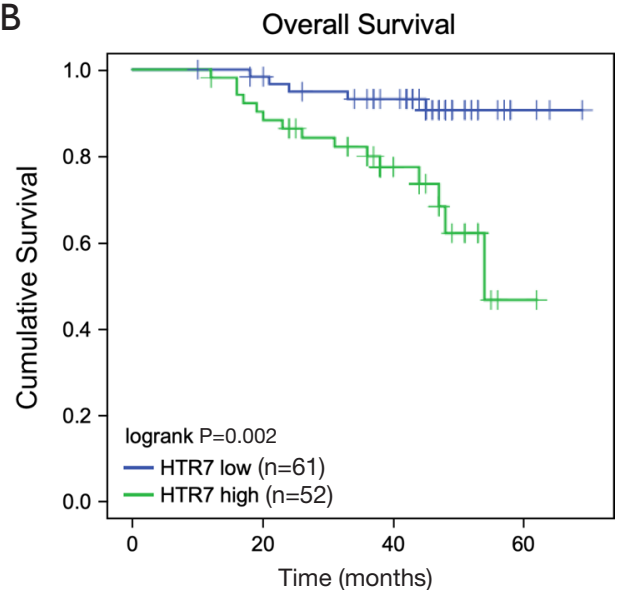

C

\begin{tabular}{|c|c|c|c|c|c|c|}
\hline & \multicolumn{3}{|c|}{ Univariate analysis } & \multicolumn{3}{|c|}{ Multivariate analysis } \\
\hline & $\begin{array}{c}\text { No. } \\
\text { patients }\end{array}$ & $P$ & $\begin{array}{l}\text { Relative } \\
\text { risk }\end{array}$ & $P$ & $\begin{array}{l}\text { Relative } \\
\text { risk }\end{array}$ & $\begin{array}{l}95 \% \text { confidence } \\
\text { interval }\end{array}$ \\
\hline \multicolumn{7}{|l|}{ Relapse } \\
\hline Yes & 35 & 0.005 & 3.596 & 0.017 & 2.966 & $1.214-7.248$ \\
\hline No & 78 & & & & & \\
\hline \multicolumn{7}{|c|}{ Distant metastasis } \\
\hline Yes & 10 & 0.002 & 5.081 & 0.068 & 2.732 & $0.930-8.026$ \\
\hline No & 103 & & & & & \\
\hline \multicolumn{7}{|c|}{ Expression of HTR7 } \\
\hline Low expression & 61 & 0.004 & 4.496 & 0.030 & 3.262 & $1.118-9.517$ \\
\hline High expression & 52 & & & & & \\
\hline
\end{tabular}

Figure 2 5-hydroxytryptamine receptor 7 (HTR7) is an independent predictive factor for the prognosis of patients with laryngeal cancer. (A) Immunohistochemical representation of low and high HTR7 expressions. (B) Overall survival analysis for HTR7 expression and patient survival time. (C) Univariate and multivariate analyses of relapse, distant metastasis, and HTR7 expression in patients with laryngeal cancer using the Cox regression analysis. Error bars represent the mean $\pm \mathrm{STDEV}$. ${ }^{*} \mathrm{P}<0.05$.

patients with laryngeal cancer using log-rank test and multivariable Cox proportional hazard regression analysis. Immunohistochemistry was used to determine HTR7 expression in the cohort of 113 patients with laryngeal cancer (Figure 2A). Kaplan-Meier survival analysis showed that patients with low HTR7 expression had longer survival than those with high HTR7 expression (Figure 2B). To identify independent prognostic factors for the survival of patients with laryngeal cancer, univariate and multivariate Cox regression models were used, which showed that relapse, distant metastasis, and HTR7 expression were significant prognostic predictors for the overall survival of patients with laryngeal cancer. Relapse and HTR7 expression were identified as independent predictive factors for the prognosis of patients with laryngeal cancer (Figure 2C). Taken together, these results showed that high HTR7 expression was a significant independent prognostic factor for poor prognosis in laryngeal cancer, and could be used as a biomarker for prognosis in patients with laryngeal cancer.

\section{HTR7 promotes laryngeal cancer proliferation in vitro}

To determine the function of HTR7 in laryngeal cancer progression, we used GSEA to analyze the relationship between HTR7 expression and the expression of key genes associated with tumor proliferation; HTR7 expression was 


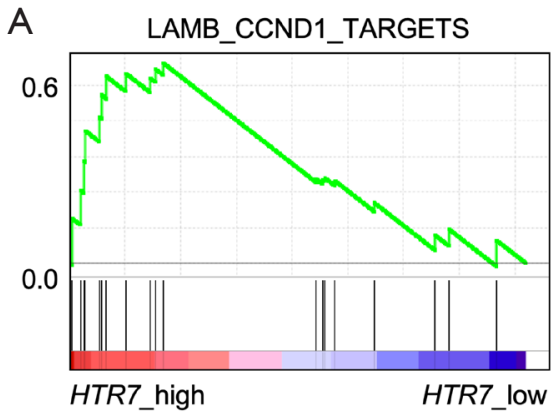

ES $=0.563 \quad \mathrm{NES}=1.635$

$\mathrm{P}=0.020 \quad \mathrm{FDR}=0.247$

D

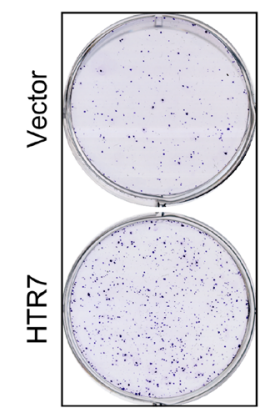

$\mathrm{FaDu}$
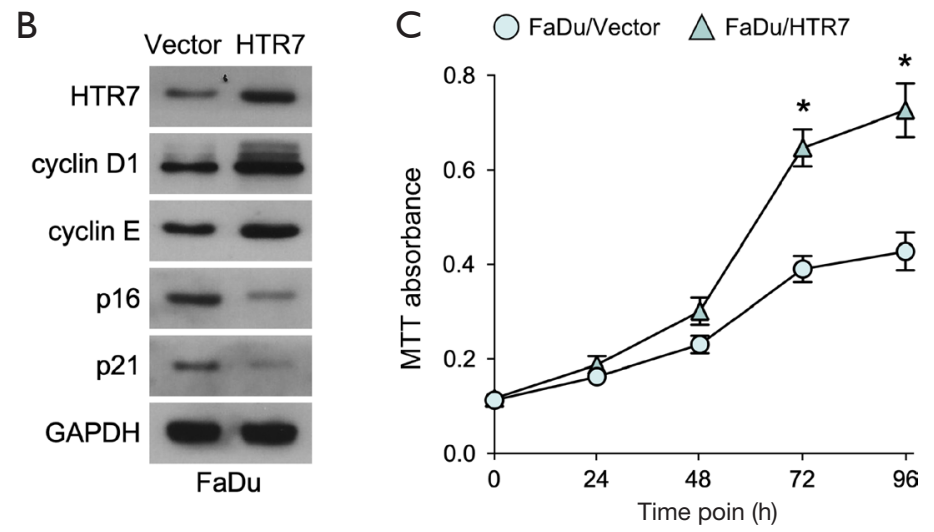

E
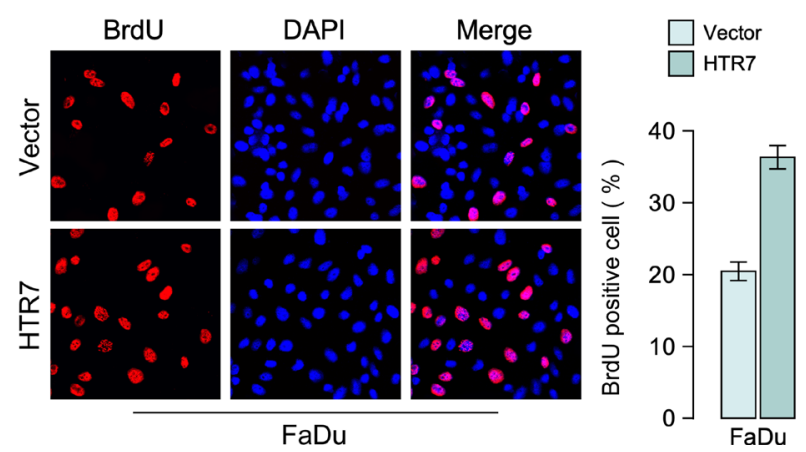

Figure 3 5-hydroxytryptamine receptor 7 (HTR7) overexpression promotes laryngeal cancer proliferation. (A) Gene Set Enrichment Analysis of the relationship between HTR7 expression and CCND1 expression. (B) Western blot analysis of cyclin D1, cyclin E, p16, and p21 levels when HTR7 was overexpressed in FaDu cells. GAPDH was used as the loading control. (C) 3-(4,5-Dimethylthiazol-2-yl)-2,5diphenyltetrazolium bromide assay of the effect of HTR7 overexpression on laryngeal cancer proliferation. (D) Colony formation assay of the effect of HTR7 overexpression on laryngeal cancer proliferation using crystal violet staining. Microscope magnification 200x. (E) BrdU incorporation assay of the effect of HTR7 overexpression on laryngeal cancer proliferation. Error bars represent the mean \pm STDEV. ${ }^{*} \mathrm{P}<0.05$.

found to be positively correlated with CCND1 expression (Figure 3A). CCND1 is a key factor for G1/S transition, and accelerates cell cycle progression $(11,12)$. Cyclin E promotes the cell cycle, and p21 and p16 inhibit the cell cycle (13-15). We first determined the effect of HRT7 level on laryngeal cancer cells, MTT analysis showed TU212 with high HTR7 level had higher proliferation rate than $\mathrm{FaDu}$ with low HTR7 level (Figure S1). We overexpressed HTR7 in the FaDu cell line, which has low HTR7 expression, and knocked down HTR7 expression in TU212, which has high HTR7 expression. Western blot analysis showed that HTR7 overexpression increased cyclin D1 and cyclin E levels and reduced p16 and p21 levels (Figure 3B). The MTT assay showed that HTR7 overexpression significantly promoted cell proliferation (Figure 3C). Colony formation assays showed that HTR7 overexpression increased cell proliferation (Figure 3D) and BrdU incorporation assays revealed that HTR7 overexpression promoted cell proliferation (Figure 3E). While HTR7 knockdown reduced cyclin $\mathrm{D}$ and cyclin $\mathrm{E}$ levels and increased p21 and p16 levels (Figure 4A), moreover cell proliferation was significantly inhibited (Figure $4 B, C, D$ ). These results suggested that HTR7 promoted the proliferation of laryngeal cancer.

\section{HTR 7 promotes laryngeal cancer growth in vivo}

To confirm these results, we used soft agar growth assays 
A

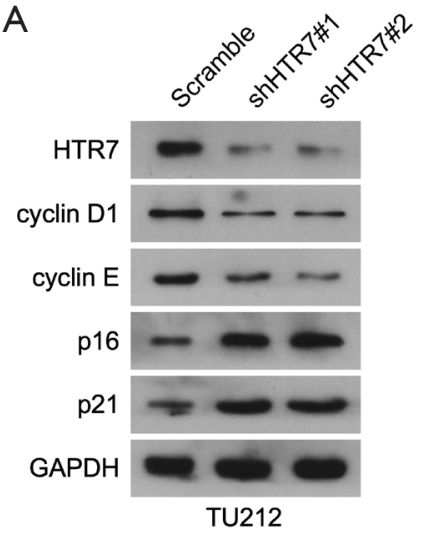

C

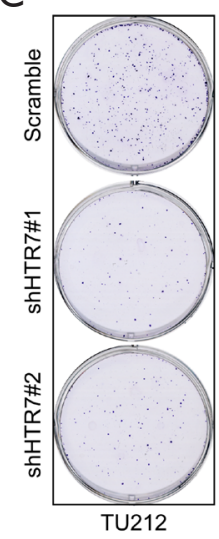

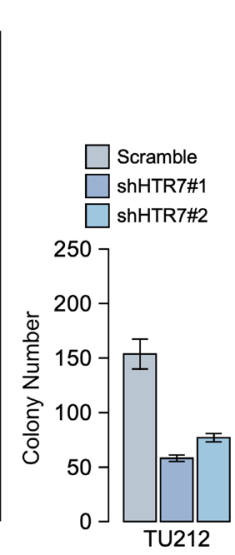

B

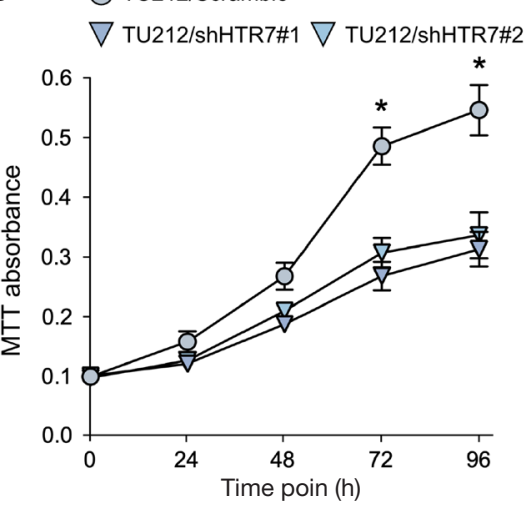

D

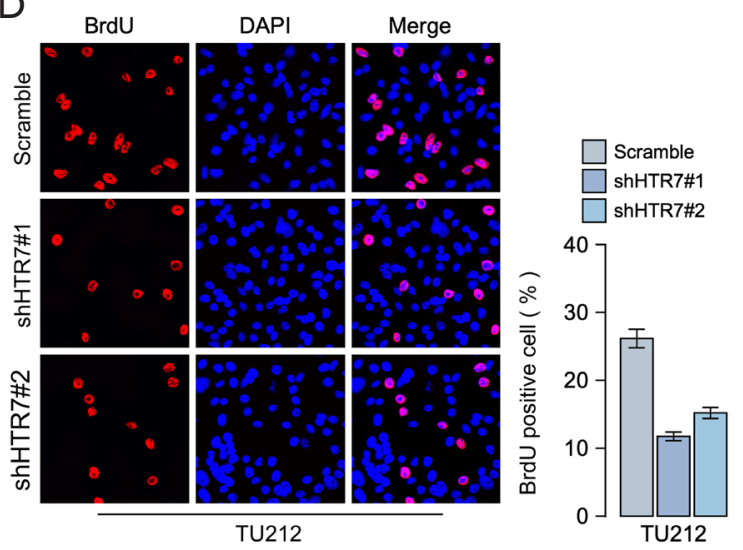

Figure 4 5-hydroxytryptamine receptor 7 (HTR7) knockdown inhibits laryngeal cancer proliferation. (A) Western blotting analysis of cyclin D1, cyclin E, p16, and p21 levels when HTR7 was knocked down in TU212 cells. GAPDH was used as the loading control. (B) 3-(4,5-Dimethylthiazol-2-yl)-2,5-diphenyltetrazolium bromide assay of the effect of HTR7 knockdown on laryngeal cancer proliferation. (C) Colony formation assay of the effect of HTR7 knockdown on laryngeal cancer proliferation using crystal violet staining. Microscope magnification 200×. (D) BrdU incorporation assay of the effect of HTR7 knockdown on laryngeal cancer proliferation. Error bars represent the mean \pm STDEV. ${ }^{*} \mathrm{P}<0.05$.

and a mouse model to determine the role of HTR7 in laryngeal cancer growth. Soft agar growth assays demonstrated that HTR7 overexpression significantly promoted tumor anchorage-independent growth, while HTR7 knockdown significantly inhibited tumor anchorageindependent growth (Figure $5 A$ ). The mouse model revealed that HTR7 overexpression significantly increased the tumor volume, while HTR7 knockdown significantly reduced the tumor volume (Figure 5B). These results suggested that HTR7 promoted laryngeal cancer growth.

\section{HTR 7 promotes laryngeal cancer growth by activating the PI3K/AKT pathway}

To determine the regulatory mechanism of HTR7 in laryngeal cancer growth, we used GSEA to analyze the signaling pathways regulated by HTR7 and found that HTR7 expression levels were positively correlated with those of PI3K/AKT pathway targets (Figure 6A). Luciferase reporter assays showed that HTR7 overexpression significantly increased luciferase activity, while HTR7 knockdown significantly inhibited luciferase activity, suggesting that HTR7 increased PI3K/AKT pathway activity (Figure 6B). Western blot assays showed that HTR7 overexpression increased the level of phosphorylated AKT, while HTR7 knockdown reduced the level of phosphorylated AKT (Figure 6C), suggesting that HTR7 activated the AKT pathway. BCL2 like 1 (BCL2L1), BCL2 related protein A1 (BCL2A1), Baculoviral IAP repeat containing 5 (BIRC5), BCL2 apoptosis regulator (BCL2), X-linked inhibitor of apoptosis (XIAP), cyclin E2 (CCNE2), cyclin D2 (CCND2), cyclin dependent kinase 2 (CDK2), cyclin dependent kinase 
A
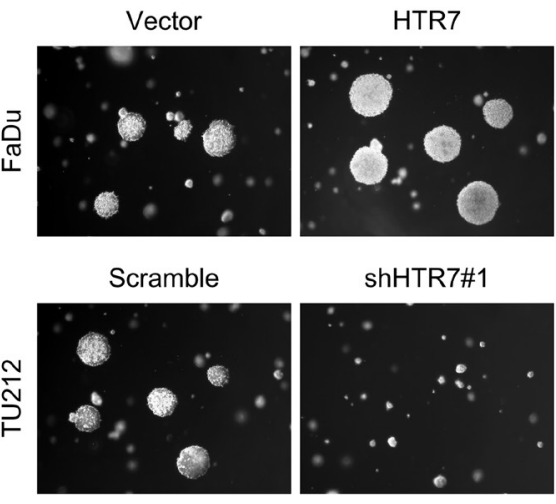

shHTR7\#1 shHTR7\#2
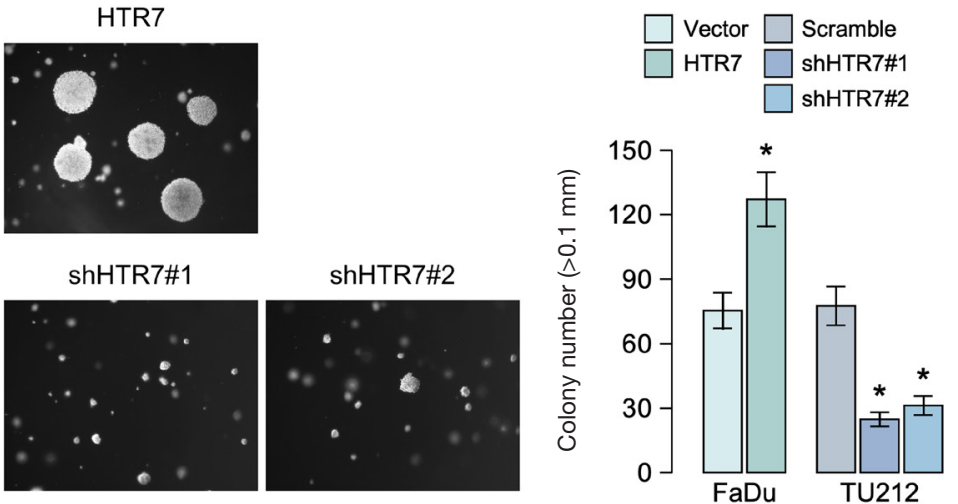

B
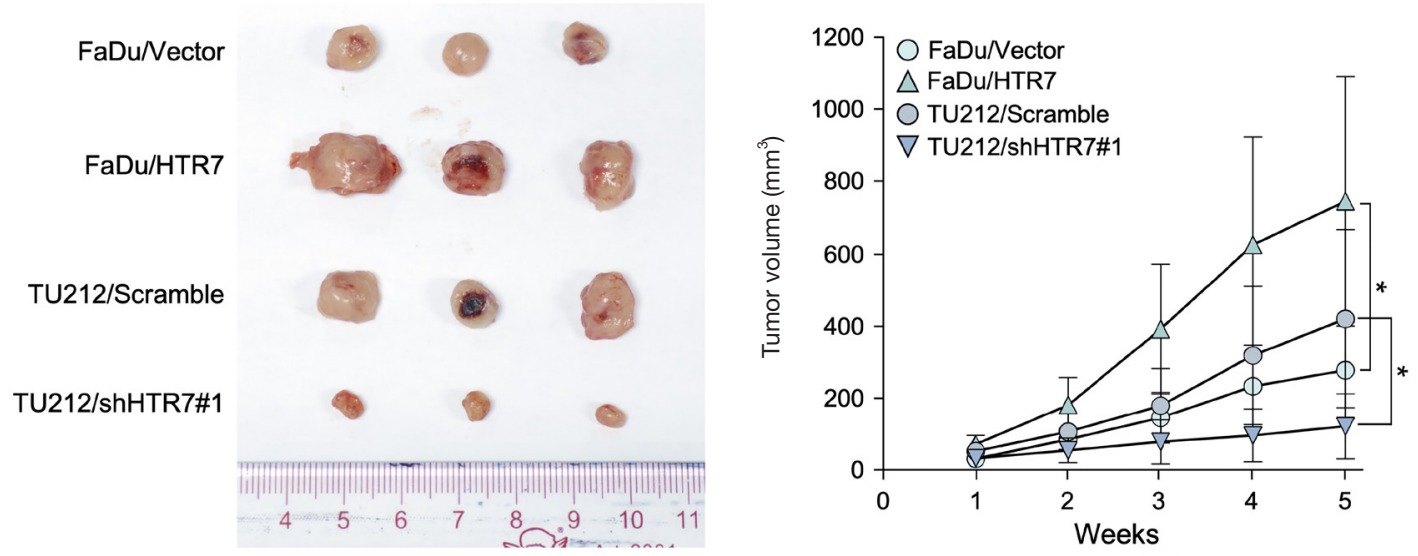

Figure 5 5-hydroxytryptamine receptor 7 (HTR7) promotes laryngeal cancer growth. (A) Soft agar growth analysis of the effect of HTR7 overexpression or knockdown on cell growth. The colony size was determined using an ocular micrometer and colonies $>0.1$ mm in diameter were counted through a bright-field microscope under $\times 400$ magnification. Representational soft agar growth assay are shown on the left; statistical analysis is shown on the right. (B) Animal model analysis of the effect of HTR7 overexpression or knockdown on cell growth. Representational soft agar growth assay are shown on the left; tumor volume analysis is shown on the right. Error bars represent the mean \pm STDEV. ${ }^{*} \mathrm{P}<0.05$.

4 (CDK4), and BCL2 associated agonist of cell death (BAD) are targets of AKT and are associated with tumor proliferation and the inhibition of apoptosis $(16,17)$. qRTPCR analysis showed that HTR7 overexpression promoted their expression, while HTR7 knockdown inhibited their expression (Figure 6D), which supported the view that HTR7 activated the PI3K/AKT pathway.

To understand whether HTR7 promoted laryngeal cancer growth by activating the AKT pathway, we inhibited PI3K/ AKT pathway activation using either small interfering RNAs targeting AKT or the AKT pathway inhibitor, perifosine, in HTR7-overexpressing laryngeal cancer cells (18). Colony formation assays showed that the inhibition of the PI3K/AKT pathway significantly inhibited cell proliferation (Figure $7 A$ ), which was confirmed using soft agar growth assays (Figure 7B). These findings indicated that HTR7 promoted laryngeal cancer growth by activating the PI3K/ AKT pathway.

We confirmed these results using clinical samples. Western blot assays showed that laryngeal cancer tissues with high HTR7 expression had high p-AKT levels, while laryngeal cancer tissues with low HTR7 expression had low p-AKT levels, and the expression of HTR7 and p-AKT levels were positively correlated (Figure 7C), suggesting that HTR7 activated the PI3K/AKT pathway in clinical samples.

\section{Discussion}

In the present study, we found that HTR7 was significantly 
A

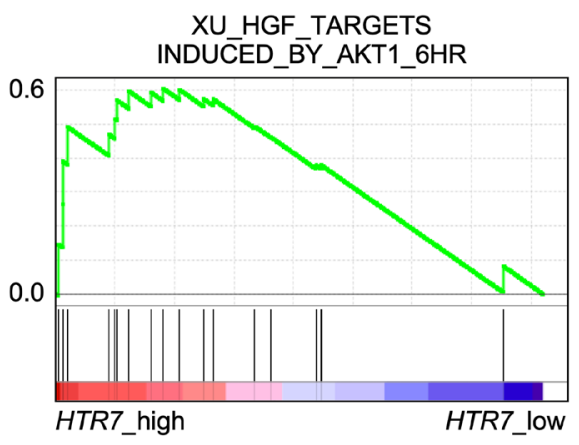

ES $=0.604 \quad \mathrm{NES}=1.524$

$\mathrm{P}=0.049 \quad \mathrm{FDR}=0.246$

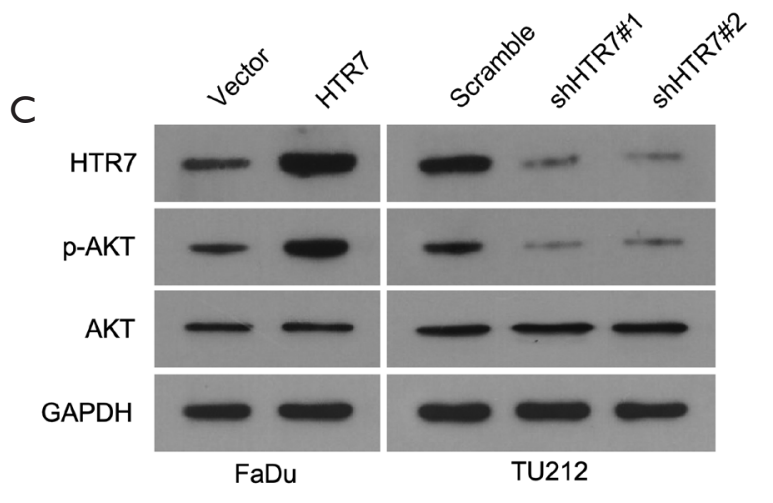

B
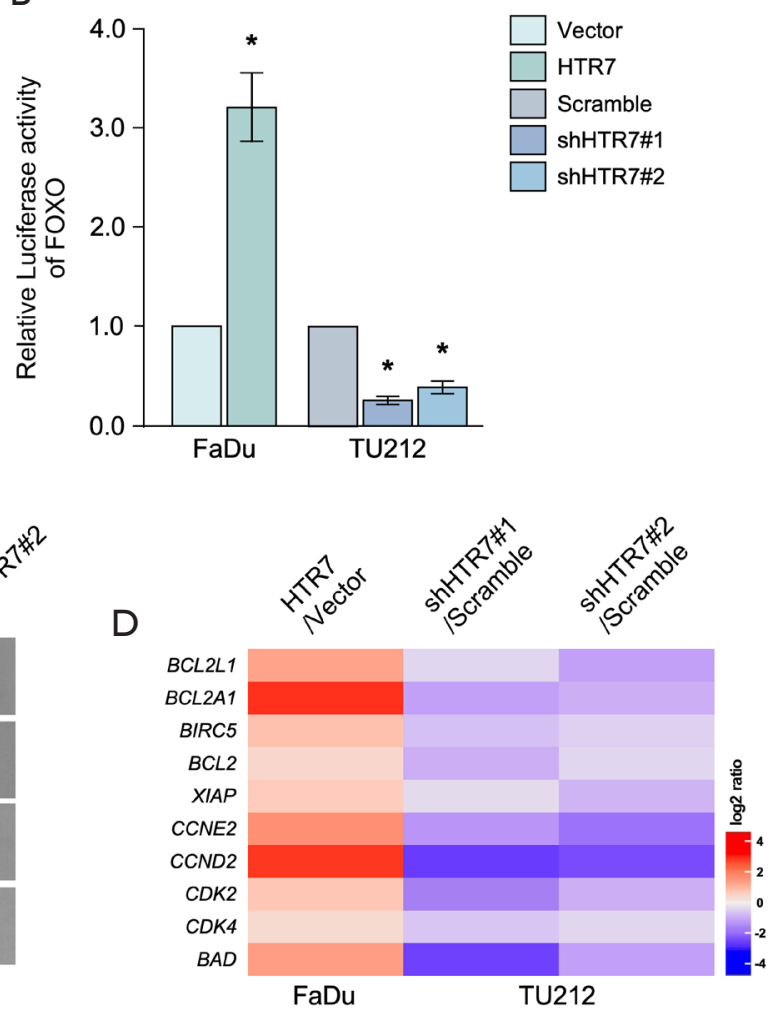

Figure 6 5-hydroxytryptamine receptor 7 (HTR7) activated the protein kinase B (AKT) pathway. (A) Gene Set Enrichment Analysis of the correlation between HTR7 expression and the expression of AKT pathway target genes. (B) Luciferase reporter analysis of FOXO transcription activity after HTR7 overexpression or knockdown. (C) Western blot analysis of AKT, p-AKT, and HTR7 levels after HTR7 overexpression or knockdown; GAPDH was used as the loading control. (D) Quantitative reverse transcription polymerase chain reaction analysis of BCL2L1, BCL2A1, BIRC5, BCL2, XIAP, CCNE2, CCND2, CDK2, CDK4, and BAD expression after HTR7 overexpression or knockdown; results are shown using a heatmap. Error bars represent the mean \pm STDEV. ${ }^{*} \mathrm{P}<0.05$.

upregulated in laryngeal cancer cells and tissues, and patients with high HTR7 expression had a shorter survival time. HTR7 was an independent predictive factor for the prognosis of patients with laryngeal squamous cell cancer. Functional assays showed that HTR7 overexpression promoted laryngeal cancer proliferation and growth. Mechanism analysis showed that HTR7 promoted laryngeal cancer proliferation and growth by activating the AKT pathway.

The PI3K/AKT pathway has been previously found to be associated with laryngeal cancer, and the phosphorylation of AKT is a marker of PI3K/AKT activation. AKT phosphorylation is associated with treatment failure of head and neck cancer, and the inhibition of AKT increases radiosensitivity (19). Many genes and non-coding RNAs have been shown to regulate laryngeal cancer progression by regulating the PI3K/AKT pathway. For example, miR132 promotes laryngeal cancer proliferation and growth by targeting FOXO1, which activates the PI3K/AKT pathway (20). TRA2 $\beta$ is associated with poor differentiation, lymph node metastasis, and advanced clinical stage of laryngeal cancer. It promotes proliferation, growth, invasion, and migration, and inhibits apoptosis by activating PI3K/AKT (21). Our findings also indicated that HTR7 promoted laryngeal cancer proliferation and growth through the PI3K/AKT pathway. HTR7 overexpression increased the level of phosphorylated AKT, while HTR7 knockdown reduced the level of phosphorylated AKT. Luciferase reporter analyses suggested that HTR7 overexpression increased FOXO transcription factor-mediated transcription. The expression of the PI3K/AKT pathway target gene also increased, further demonstrating that HTR7 activated the PI3K/ 

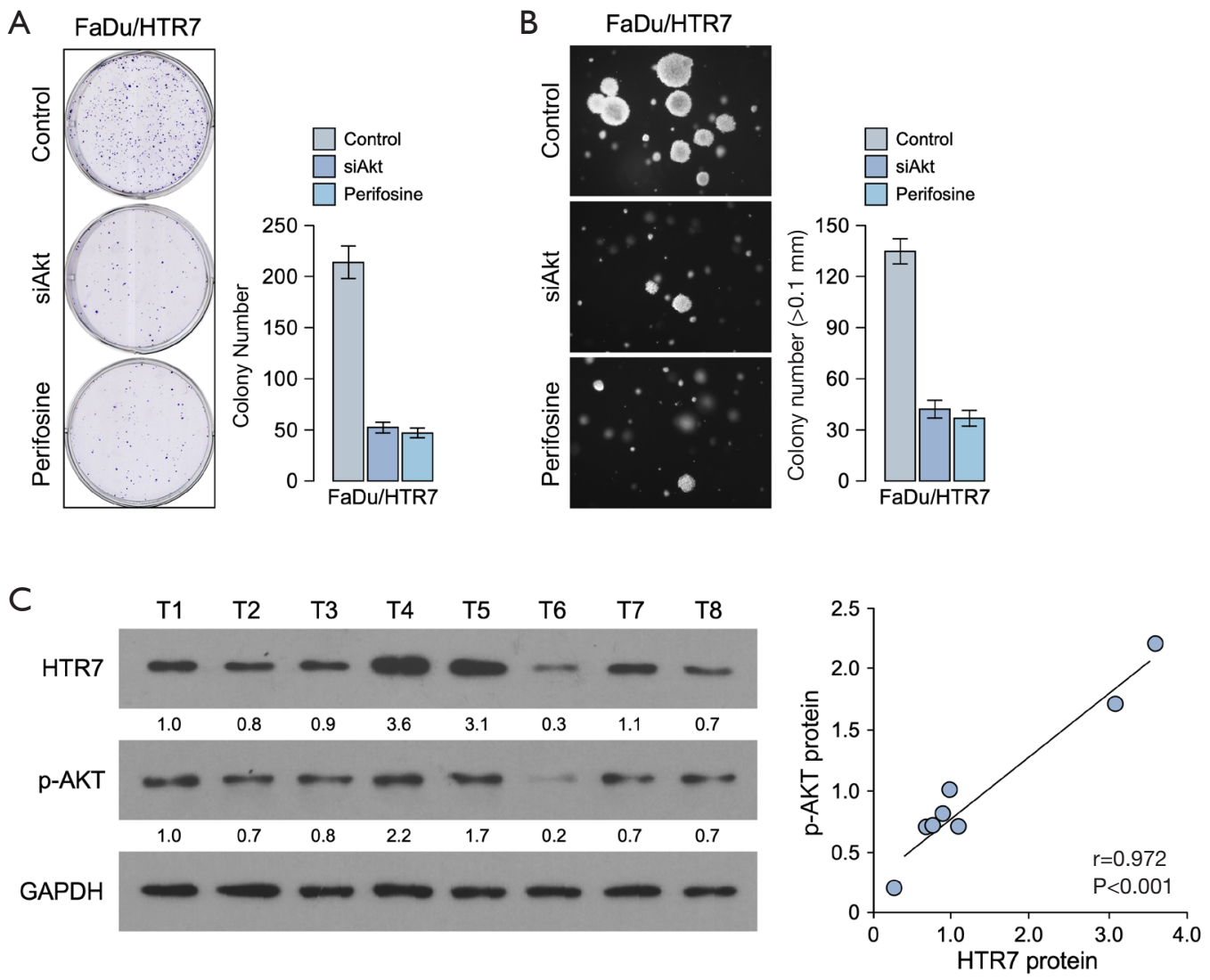

Figure 7 5-hydroxytryptamine receptor 7 (HTR7) promotes laryngeal cancer growth by activating the protein kinase B (AKT) pathway. (A) Colony formation assay of the effect on cell growth of inhibition of the AKT pathway in HTR7-overexpressing cells. (B) Soft agar growth analysis of the effect on cell growth of inhibition of the AKT pathway in HTR7-overexpressing cells. The colony size was determined using an ocular micrometer and colonies $>0.1 \mathrm{~mm}$ in diameter were counted through a bright-field microscope under $\times 400 \mathrm{magnification}$. Representative soft agar growth assay is shown on the left; statistical analysis is shown on the right. (C) Western blot analysis of HTR7 and p-AKT levels in laryngeal cancer tissues, and the statistical analysis of the relationship between HTR7 and p-AKT levels. GAPDH was used as the loading control. Error bars represent the mean $\pm \mathrm{STDEV}$. ${ }^{*} \mathrm{P}<0.05$.

AKT pathway. The inhibition of the PI3K/AKT pathway in HTR7-overexpressing cells inhibited cell proliferation and growth, suggesting that HTR7 promoted laryngeal cancer proliferation and growth by activating the PI3K/AKT pathway.

To the best of our knowledge, the role of HTR7 in tumor progression has not been studied until now. HTR7 belongs to the GPCR family, which is one of the most studied pharmacological targets because of its diverse biological functions and druggable sites. GPCRs represent about $34 \%$ of drugs approved by the US Food and Drug Administration, and about $27 \%$ in terms of sales (22-24), suggesting that HTR7 might be easily druggable to treat laryngeal cancer. In summary, HTR7 promotes laryngeal cancer growth by activating the PI3K/AKT pathway, providing a new target for laryngeal cancer prognosis and therapy.

\section{Acknowledgments}

Funding: This work was financially supported by the Natural Science Foundation of Guangdong Province, China (Grant No. 2019A1515011678).

\section{Footnote}

Reporting Checklist: The authors have completed the ARRIVE reporting checklist. Available at http://dx.doi. 
org/10.21037/atm-21-1069

Data Sharing Statement: Available at http://dx.doi. org/10.21037/atm-21-1069

Conflicts of Interest: All authors have completed the ICMJE uniform disclosure form (available at http://dx.doi. org/10.21037/atm-21-1069). The authors have no conflicts of interest to declare.

Ethical Statement: The authors are accountable for all aspects of the work in ensuring that questions related to the accuracy or integrity of any part of the work are appropriately investigated and resolved. The study was conducted in accordance with the Declaration of Helsinki (as revised in 2013). The Ethics Committee of Guangdong Provincial People's Hospital and Guangdong Academy of Medical Sciences approved all collections. All patients provided written informed consent. All animal experiments were performed in accordance with the guidelines for animal care and under the protocols approved by the Institutional Animal Care and Use Committee of the Guangdong Provincial People's Hospital and Guangdong Academy of Medical Sciences.

Open Access Statement: This is an Open Access article distributed in accordance with the Creative Commons Attribution-NonCommercial-NoDerivs 4.0 International License (CC BY-NC-ND 4.0), which permits the noncommercial replication and distribution of the article with the strict proviso that no changes or edits are made and the original work is properly cited (including links to both the formal publication through the relevant DOI and the license). See: https://creativecommons.org/licenses/by-nc-nd/4.0/.

\section{References}

1. Megwalu UC, Sikora AG. Survival outcomes in advanced laryngeal cancer. JAMA Otolaryngol Head Neck Surg 2014;140:855-60.

2. Steuer CE, El-Deiry M, Parks JR, et al. An update on larynx cancer. CA Cancer J Clin 2017;67:31-50.

3. Hemmings BA, Restuccia DF. Pi3k-pkb/akt pathway. Cold Spring Harb Perspect Biol 2012;4:a011189.

4. Mayer IA, Arteaga CL. The pi3k/akt pathway as a target for cancer treatment. Annu Rev Med 2016;67:11-28.

5. Manning BD, Toker A. Akt/pkb signaling: Navigating the network. Cell 2017;169:381-405.
6. Matthys A, Haegeman G, Van Craenenbroeck K, et al. Role of the 5-ht7 receptor in the central nervous system: From current status to future perspectives. Mol Neurobiol 2011;43:228-53.

7. Laenen K, Haegeman G, Vanhoenacker P. Structure of the human 5-ht7 receptor gene and characterization of its promoter region. Gene 2007;391:252-63.

8. Di Pilato P, Niso M, Adriani W, et al. Selective agonists for serotonin 7 (5-ht7) receptor and their applications in preclinical models: An overview. Rev Neurosci 2014;25:401-15.

9. Ikeda M, Iwata N, Kitajima T, et al. Positive association of the serotonin 5-ht7 receptor gene with schizophrenia in a japanese population. Neuropsychopharmacology 2006;31:866-71.

10. Erdmann J, Nothen MM, Shimron-Abarbanell D, et al. The human serotonin 7 (5-ht7) receptor gene: Genomic organization and systematic mutation screening in schizophrenia and bipolar affective disorder. Mol Psychiatry 1996;1:392-7.

11. Qie S, Diehl JA. Cyclin d1, cancer progression, and opportunities in cancer treatment. J Mol Med (Berl) 2016;94:1313-26.

12. Sanchez G, Delattre O, Auboeuf D, et al. Coupled alteration of transcription and splicing by a single oncogene: Boosting the effect on cyclin $\mathrm{d} 1$ activity. Cell Cycle 2008;7:2299-305.

13. Ishidate T, Elewa A, Kim S, et al. Divide and differentiate: $\mathrm{Cdk} /$ cyclins and the art of development. Cell Cycle 2014;13:1384-91.

14. Schafer KA. The cell cycle: A review. Vet Pathol 1998; 35:461-78.

15. He S, Sharpless NE. Senescence in health and disease. Cell 2017;169:1000-11.

16. Nozhat Z, Hedayati M. Pi3k/akt pathway and its mediators in thyroid carcinomas. Mol Diagn Ther 2016;20:13-26.

17. Alzahrani AS. Pi3k/akt/mtor inhibitors in cancer: At the bench and bedside. Semin Cancer Biol 2019;59:125-32.

18. Patel V, Lahusen T, Sy T, et al. Perifosine, a novel alkylphospholipid, induces p21(waf1) expression in squamous carcinoma cells through a p53-independent pathway, leading to loss in cyclin-dependent kinase activity and cell cycle arrest. Cancer Res 2002;62:1401-9.

19. Gupta AK, McKenna WG, Weber CN, et al. Local recurrence in head and neck cancer: Relationship to radiation resistance and signal transduction. Clin Cancer Res 2002;8:885-92.

20. Lian R, Lu B, Jiao L, et al. Mir-132 plays an oncogenic 
Page 12 of 12

role in laryngeal squamous cell carcinoma by targeting foxo1 and activating the pi3k/akt pathway. Eur J Pharmacol 2016;792:1-6.

21. Ni HS, Hu SQ, Chen X, et al. Tra2beta silencing suppresses cell proliferation in laryngeal squamous cell carcinoma via inhibiting pi3k/akt signaling. Laryngoscope 2019;129:E318-28.

22. Chiba T, Kimura S, Takahashi K, et al. Serotonin regulates beta-casein expression via 5-ht7 receptors in human mammary epithelial mcf-12a cells. Biol Pharm Bull

Cite this article as: Sheng $\mathrm{X}, \mathrm{Liu} \mathrm{W}, \mathrm{Lu} \mathrm{Z}, \mathrm{Xu}$ M, Li R, Zhong R, Li Y, Liu T, Zhang S. HTR7 promotes laryngeal cancer growth through PI3K/AKT pathway activation. Ann Transl Med 2021;9(10):840. doi: 10.21037/atm-21-1069

\section{Sheng et al. HTR7-PI3K-AKT axis promotes laryngeal cancer}

2015;38:448-53.

23. Bassilana F, Nash M, Ludwig MG. Adhesion g proteincoupled receptors: Opportunities for drug discovery. Nat Rev Drug Discov 2019;18:869-84.

24. Hauser AS, Attwood MM, Rask-Andersen M, et al. Trends in gpcr drug discovery: New agents, targets and indications. Nat Rev Drug Discov 2017;16:829-42.

(English Language Editor: R. Scott) 


\section{Supplementary}

Table S1 Clinicopathological characteristics of studied patients and expression of 5-hydroxytryptamine receptor 7 (HTR7) in laryngeal cancer

\begin{tabular}{|c|c|}
\hline Characteristics & No. cases \\
\hline \multicolumn{2}{|l|}{ Age (years) } \\
\hline$<55$ & 27 \\
\hline$\geq 55$ & 86 \\
\hline \multicolumn{2}{|l|}{ Sex } \\
\hline Male & 106 \\
\hline Female & 7 \\
\hline \multicolumn{2}{|l|}{ T stage } \\
\hline $\mathrm{T} 1$ & 52 \\
\hline $\mathrm{T} 2$ & 7 \\
\hline T3 & 28 \\
\hline T4 & 26 \\
\hline \multicolumn{2}{|l|}{$\mathrm{N}$ stage } \\
\hline NO & 85 \\
\hline N1 & 20 \\
\hline N2 & 7 \\
\hline N3 & 1 \\
\hline \multicolumn{2}{|l|}{ M stage } \\
\hline MO & 103 \\
\hline M1 & 10 \\
\hline \multicolumn{2}{|l|}{ Clinical stage } \\
\hline I & 38 \\
\hline II & 16 \\
\hline III & 27 \\
\hline IV & 32 \\
\hline \multicolumn{2}{|l|}{ Thyroid cartilage invasion } \\
\hline Yes & 25 \\
\hline No & 88 \\
\hline \multicolumn{2}{|l|}{ Status (at follow-up) } \\
\hline Alive & 94 \\
\hline Death due to laryngeal cancer & 19 \\
\hline Death due to other cause & 0 \\
\hline \multicolumn{2}{|l|}{ HTR7 expression } \\
\hline Negative & 1 \\
\hline Positive & 112 \\
\hline Low & 61 \\
\hline High & 52 \\
\hline
\end{tabular}

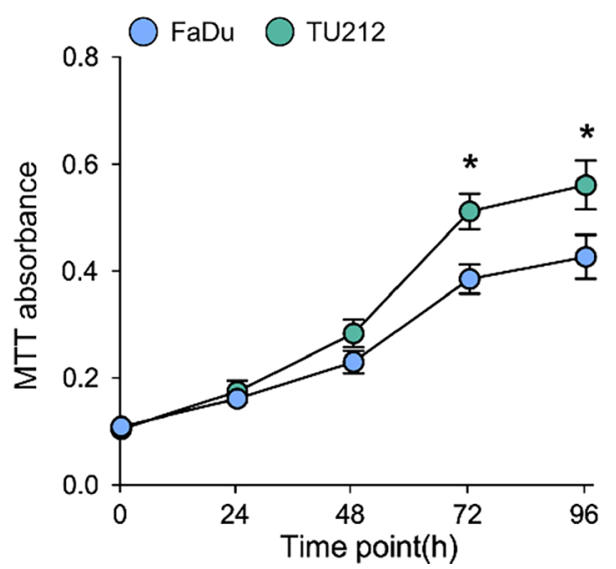

Figure S1 High 5-hydroxytryptamine receptor 7 (HTR7) expression promotes laryngeal cancer proliferation. MTT analysis of laryngeal cancer cells with different HTR7 expression. ${ }^{*} \mathrm{P}<0.05$. 